\title{
Multitasking Human Body Measurement
}

\author{
Meenakshi Amit Chaudhari \\ ${ }^{I}$ Asst Professor, Vishwatmak Om Gurudev College of Engineering, Electronics and Telecommunication dept, \\ Mumbai, India,421601,
}

Received on: 16 July, 2021

Revised on: 14 August, 2021

Published on: 16 August, 2021

\begin{abstract}
In my major project we can measure heart rate, pulse rate, blood pressure and body temperature. Heart rate reading will be displayed on LED by measuring the time between the two signal peak values and after that calculating the frequency of beats per minutes. Now a days we frequently visit to the doctor to get their vital signs measured. There is number of tools for non-invasive methods of measurement of these vital signs. The main objective of this proposed project is that to design and implement a reliable, low powered, cheap, non-intrusive, and accurate system that can be used on a regular basis and monitors the vital signs and displays the output to the LCD. This data will easily be obtained by this instrument which is wireless network. This project specifically deals with the data collection and signal conditioning of three vital signs: heart rate, blood pressure, and body temperature. Heart rate is measured through an Electrocardiogram that can obtained by attaching skin surface electrodes on the patient's wrists and fingers. Blood pressure combines the methodologies of Electrocardiography and Photo plethysmography to continuously monitor the systolic and diastolic blood pressure. Body temperature is measured inside the arms with a thermistor.
\end{abstract}

Keywords- Heart sensor, LM35, Blood pressure sensor (NPP301A), LCD. Microcontroller PIC16F877A

\section{I - INTRODUCTION}

$\mathbf{T}$ he aim of this project is to implement a tool which will measure vital sign, pressure and blood heat. during this project the most challenges are to amplifying the specified weak signal within the presence of noise from other muscles and electrical sources. Reading of rate are going to be obtained by measuring time between two signals peaks and so calculating the frequency of the peaks in units of beats per minute. This device is most useful if it's portable. this needs battery to be ready to power all of the required components also because the power output of the battery to be regulated. When the body is exerted the speed at which the guts beats will vary proportional to the number of efforts being exerted. By detecting the voltage created by the beating of the centre, its rate is often easily observed and used for variety of health purposes. This system reads, stores and analyses the centre beat rate signals repetitively in real-time. The hardware and software design are oriented into a single-chip microcontroller-based system, that's why it's compact in size. This design is chosen to be the acquisition circuit to observe temperature because it is slightly more accurate than the half-bridge circuit (after applying the offset). Also, cheaper components are wont to build this circuit, making it cost effective also as low power.

\section{II- LITERATURE REVIEW}

[1] In this paper the Technology Initiative for Disabled and Elderly People (TIDE).[2] This is based on Bluetooth technology. [3] In this paper the development and clinical evaluation of a wireless platform for health signs sensing. The sensors measure physical activity, ECG, blood oxygen saturation, temperature and respiratory rate. [4] In this paper, they proposed a design and implementation method of the family medical monitoring system based on embedded uC/OS-II and General Packet Radio Service (GPRS). [5] in this paper pain intensity measurement method based on physiological signals.[6] In this paper author discovered 


\section{International Journal of Innovations in Engineering and Science, www.ijies.net}

new technology to monitoring patient by Raspberry Pi. [7] In this paper they discuss about the diseases related to issues with blood pressure are becoming a major threat to human health.[8] In this paper technology of wireless sensor and wearable systems, including research about wireless sensor nodes, wireless sensor network, and the measurement of temperature, oxygen saturation and motion state signal.[9] this paper is based on physiological parameter monitoring systems. [10] (VRNNs) is used in it. [11] This paper describes a multitasking robotic platform for Minimally Invasive Surgery (MIS). The device is designed to be introduced through a standard trocar port.

\section{III- METHODOLOGY}

\section{Block Diagram}

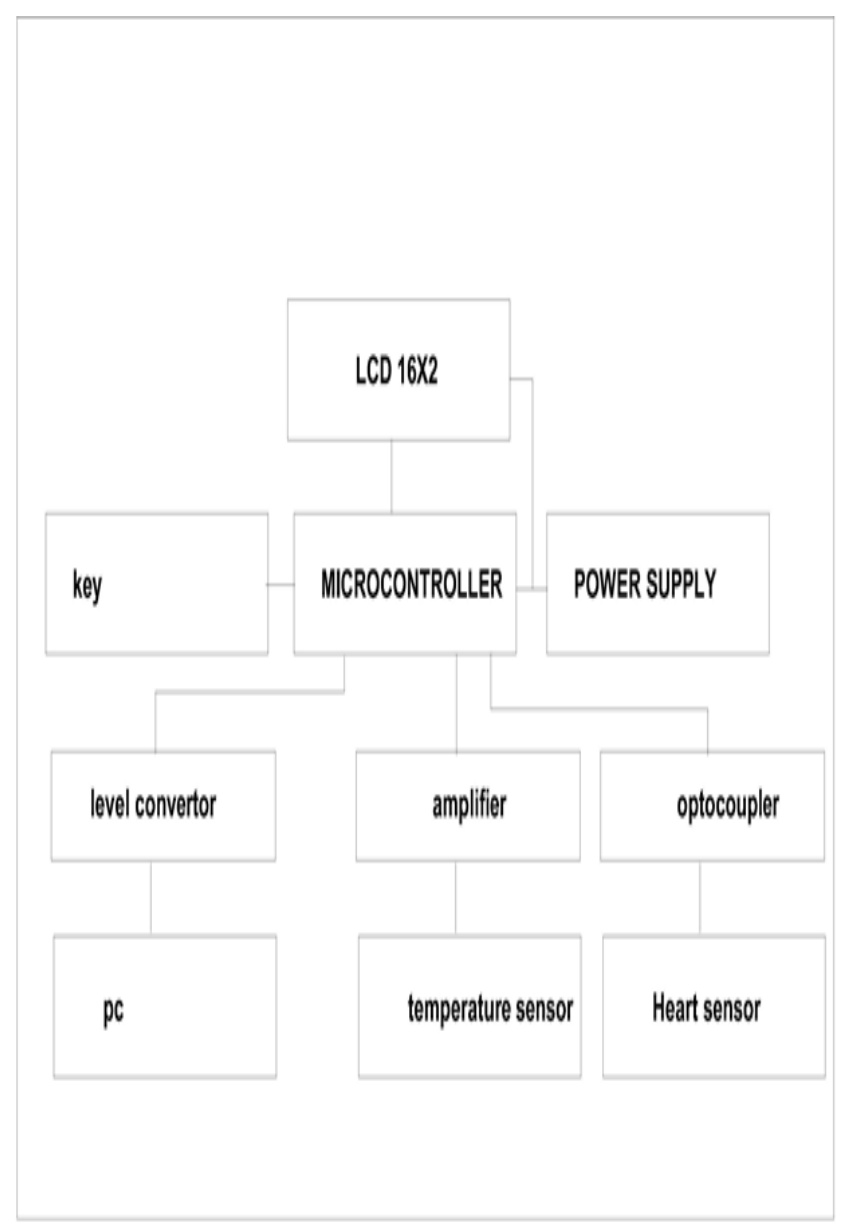

Fig 1- Block Diagram of Multitasking Human Body Measurement

\section{Block Diagram Description}

LCD 16x2: we used 16x2 LCD for display.
Amplifier: An electronic amplifier, amplifier, or amplifier is a device that increases the ability of a proof. It does this by taking energy from an influence supply and controlling the output to match the signal shape but with larger amplitude. during this sense, an amplifier modulates the output of the facility supply. Numerous varieties of electronic amplifiers are specialized to varied applications. An amplifier can check with anything from a electric circuit that uses one active component, to an entire system like a packaged audio hi-fi amplifier.

Level Convertor: This will work with a supply voltage of three. $3 \mathrm{v}$ to $5 \mathrm{v}$ making it ideal for interfacing with a PIC It derives the negative supply for the RS232 transmit data from the serial RS232 receive data line during a parasitic fashion. this suggests that the device it's connected to must use voltage levels within the RS232 specification. you cannot as an example connect two of those back-to-back since nothing is supplying the negative voltage.

Heart Sensor: Heart beat sensor is intended to allow digital output of warmth beat when a finger is placed thereon. When the center beat detector is functioning, the beat LED flashes in unison with each heartbeat. This digital output is connected to microcontroller on to measure the Beats Per Minute (BPM) rate. It works on the principle of sunshine modulation by blood flow through finger at each pulse.

Temperature Sensor: The LM35 series are precision integrated-circuit temperature sensors, whose output voltage is linearly proportional to the Celsius (Centigrade) temperature. The LM35 thus has a bonus over linear temperature sensors calibrated in ${ }^{\circ}$ Kelvin, because the user isn't required to subtract an oversized constant voltage from its output to get convenient Centigrade scaling. The LM35 doesn't require any external calibration or trimming to supply typical accuracies of $\pm 1 / 4^{\circ} \mathrm{C}$ at temperature and $\pm 3 / 4^{\circ} \mathrm{C}$ over a full -55 to $+150^{\circ} \mathrm{C}$ temperature range. Low cost is assured by trimming and calibration at the water level. The LM35's low output impedance, linear output, and precise inherent calibration make interfacing to readout or control circuitry especially easy. It are often used with single power supplies, or with plus and minus supplies. because it draws only $60 \mu \mathrm{A}$ from its supply, it's very low selfheating, but $0.1^{\circ} \mathrm{C}$ in still air. The LM35 is rated to work over a $-55^{\circ}$ to $+150^{\circ} \mathrm{C}$ temperature range, while 
Vol. 6 , No. 10, 2021, PP. $54-59$

\section{International Journal of Innovations in Engineering and Science, www.ijies.net}

the $\mathrm{LM} 35 \mathrm{C}$ is rated for $\mathrm{a}-40^{\circ}$ to $+110^{\circ} \mathrm{C}$ range $\left(-10^{\circ}\right.$ with improved accuracy). The LM35 series is on the market packaged in hermetic TO-46 transistor packages, while the LM35C, LM35CA, and LM35D also are available within the plastic TO-92 transistor package. The LM35D is additionally available in an 8-lead surface mount small outline package and plastic to-220 package.

\section{FLOWCHART}

The flowchart as shown in fig.2. describes the source code in a pictorial format.

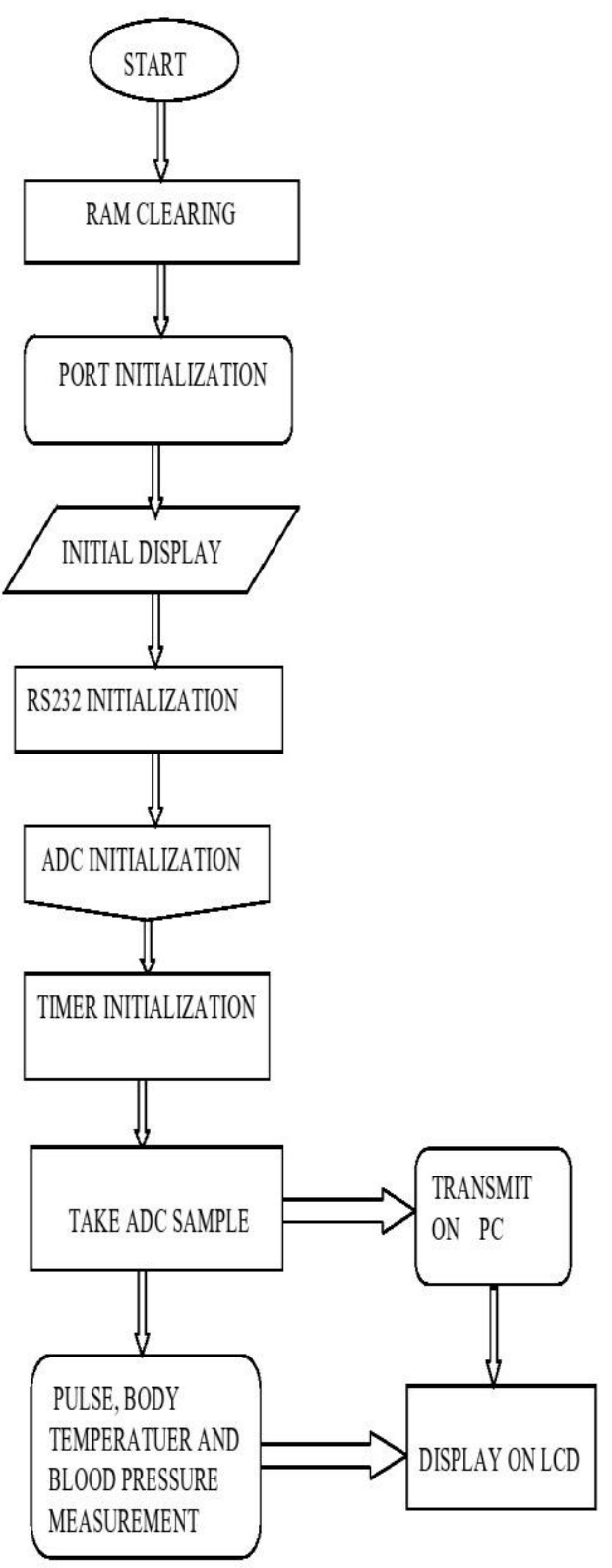

This is the circuit diagram which shows the connections of microcontroller, LCD, voltage regulator, crystal oscillator, MAX232 and switches.

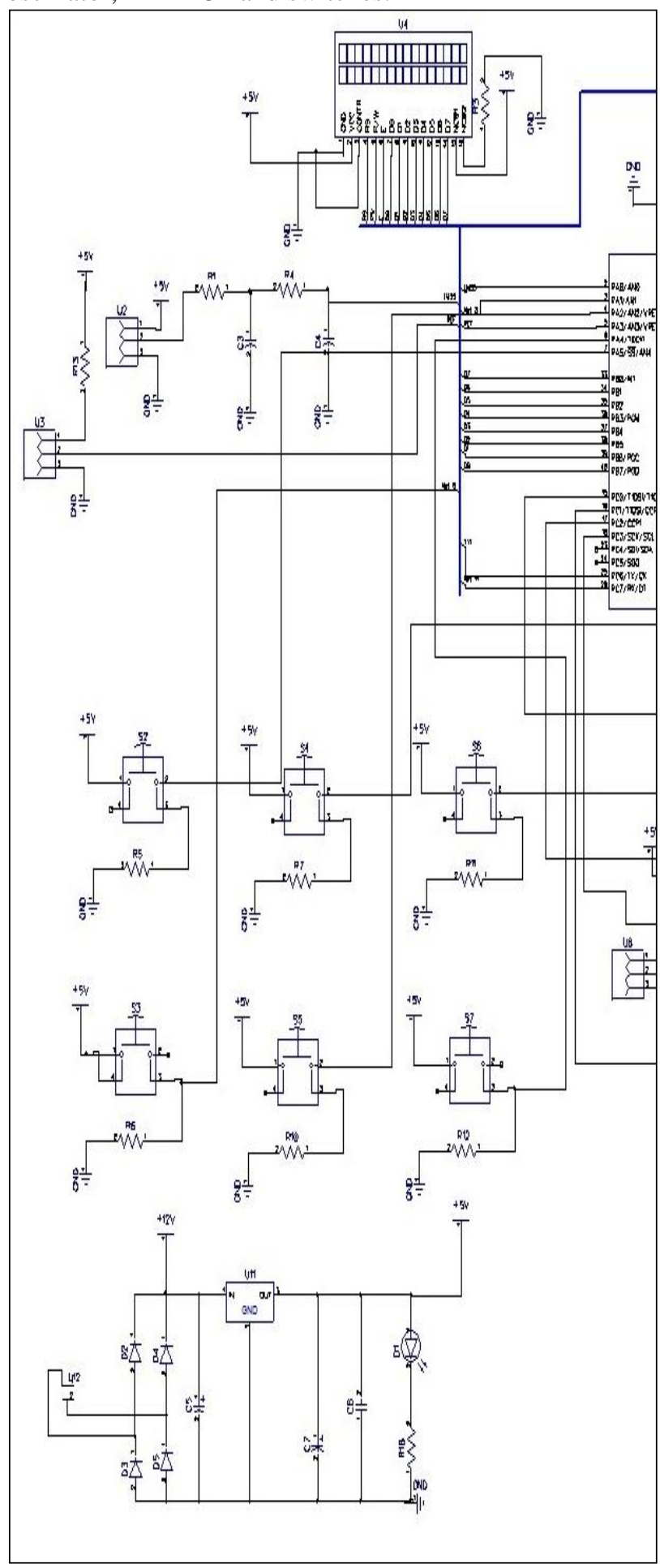

Fig 3(a) Circuit Diagram of Multitasking Human Body Measurement 


\section{International Journal of Innovations in Engineering and Science, www.ijies.net}

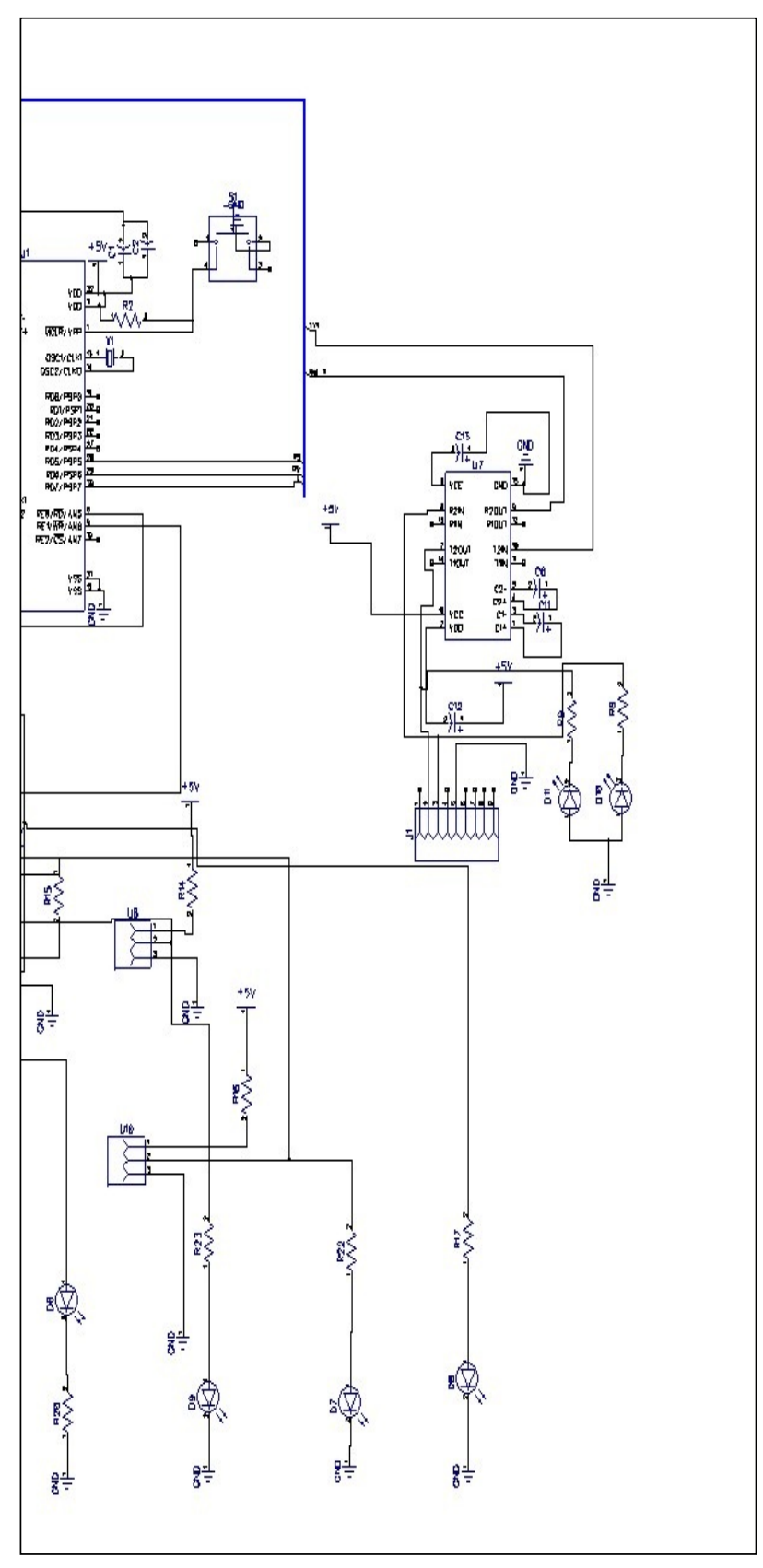

Fig 3(b) Circuit Diagram of Multitasking Human Body Measurement

\section{Component Description}

1. Push Button Switches (Reset): Both locking or latching (contacts remaining operated after the button is pressed) and unlocking (contacts release after removal of the finger) designs are available. Other design variations have multiple contacts, snap action and wiper action. In most design, state of the switch (operated or not) is set by visual observation only. Some designs have a sign light either self-contained or separate
2. MAX 323: The MAX220-MAX249 family of line drivers/receivers is meant for all EIA/TIA-232E and V.28/V.24 communications interfaces, particularly applications where $\pm 12 \mathrm{~V}$ isn't available. These parts are especially useful in battery-powered systems, since their low-power shutdown mode reduces power dissipation to but $5 \mu \mathrm{W}$. The MAX225, MAX233, MAX235, and MAX245/MAX246/MAX247 use no external components and are recommended for applications where printed circuit board space is critical.

3 Crystal Oscillator: A oscillator is an electronic oscillator circuit that uses the mechanical resonance of a vibrating crystal of piezoelectric material to make an electrical signal with an awfully precise frequency. This frequency is usually wont to keep track of your time as in quartz wrist watches, to produce a stable clock signal for digital integrated circuits, and to stabilize frequencies for radio transmitters and receivers. The foremost common style of piezoelectric resonator used is that the quartz, so oscillator circuits incorporating them became referred to as crystal oscillators, but other piezoelectric materials including polycrystalline ceramics are utilized in similar circuits.

4 Voltage Regulator: To make the overall circuit to work efficiently and error free, we start with a voltage regulator which controls the effective voltage that is being received by the microcontroller. The basic underlying thought process being that sudden voltage fluctuations or sometimes may be more voltage than is rated for the microcontroller may damage it.

5 DB 9: A D-sub contains two or more parallel rows of pins or sockets usually surrounded by a D-shaped metal shield that has mechanical support, ensures correct orientation, and should screen against electromagnetic interference. The part containing pin contacts is termed the male connector or plug, while that containing socket contacts is named the feminine connector or socket. The socket's shield fits tightly inside the plug's shield. The plug also may have screws on either side of the shield that fasten into holes within the socket.

6 PIC16F877A: The PIC16F877A CMOS FLASHbased 8-bit microcontroller is upward compatible with the PIC16C5x, PIC12Cxxx and PIC16C7x devices. It features 200 ns execution, 256 bytes of EEPROM data memory, self-programming, an ICD, 2 Comparators, 8 channels of 10-bit Analog-to-Digital (A/D) converter, to capture/compare/PWM functions, 


\section{International Journal of Innovations in Engineering and Science, www.ijies.net}

synchronous port that may be configured as either 3-wire SPI or 2-wire I2C bus and a Parallel Slave Port.

\section{IV- DESIGN}

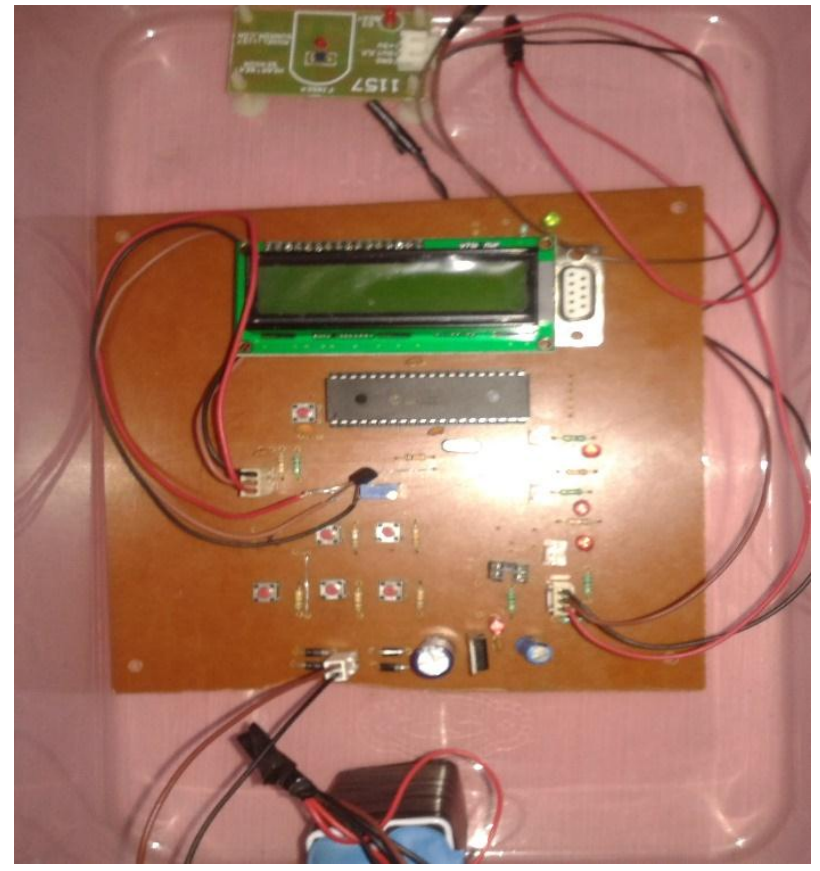

Fig.4 PCB with Components

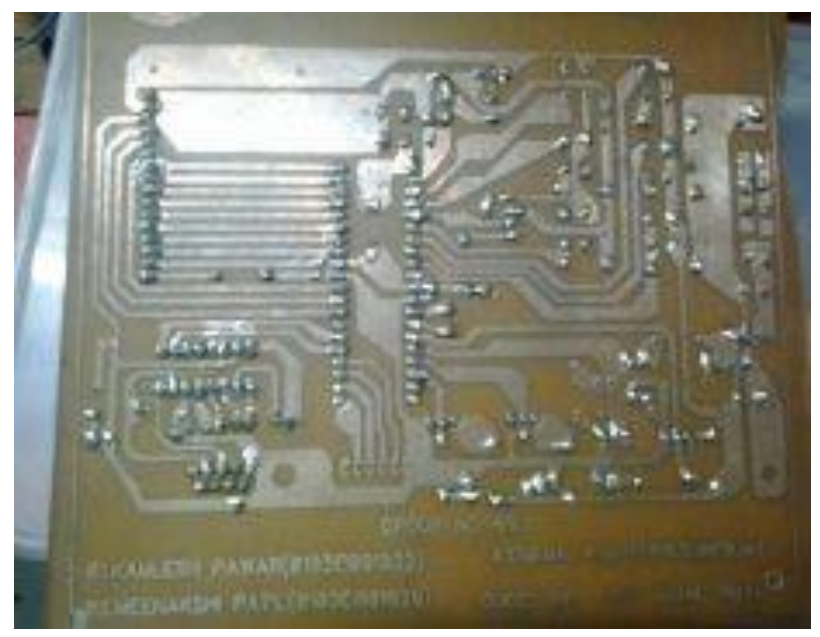

Fig 5-PCB after Etching and Soldering

\section{V- RESULT \& DISCUSSION}

The received current signals from the operational amplifier is used to derive the microcontroller. We programmed the microcontroller in such a way that it produces the output on the LCD as the rate of the heart. It calculates the rate for every 30 seconds and then multiply with 2 . As a result, the heart rate meter is able to calculate the heart rate for every 30 seconds. It is able to calculate the blood pressure within some errors. But the heart rate monitor, body temperature and blood pressure model are working and the results are when these parameters are taken from the group members: Future studies are needed to better define the relation of interruptions, and other trends of multitasking in healthcare system.

Table 1- Results

\begin{tabular}{|c|c|c|c|c|c|}
\hline \multirow[t]{2}{*}{ MEMBERS } & \multirow{2}{*}{$\begin{array}{l}\text { HEART } \\
\text { RATE }\end{array}$} & \multirow{2}{*}{\multicolumn{2}{|c|}{$\begin{array}{c}\text { BODY } \\
\text { TEMPERATURE } \\
\left(\text { in }{ }^{\circ} \mathrm{C},{ }^{\circ} \text { ) }\right.\end{array}$}} & \multicolumn{2}{|c|}{ BL00D PRESSURE } \\
\hline & & & & Systolic & Diastolic \\
\hline MEENAKSHI & 70 & $36.6^{\circ} \mathrm{C}$ & $97.9^{\circ} \mathrm{F}$ & 118 & 78 \\
\hline KAMLESH & 72 & $37^{\circ} \mathrm{C}$ & $98.6^{\circ} \mathrm{F}$ & 119 & 80 \\
\hline NIRMAL & 70 & $37.6^{\circ} \mathrm{C}$ & $99.7{ }^{\circ} \mathrm{F}$ & 117 & 76 \\
\hline
\end{tabular}

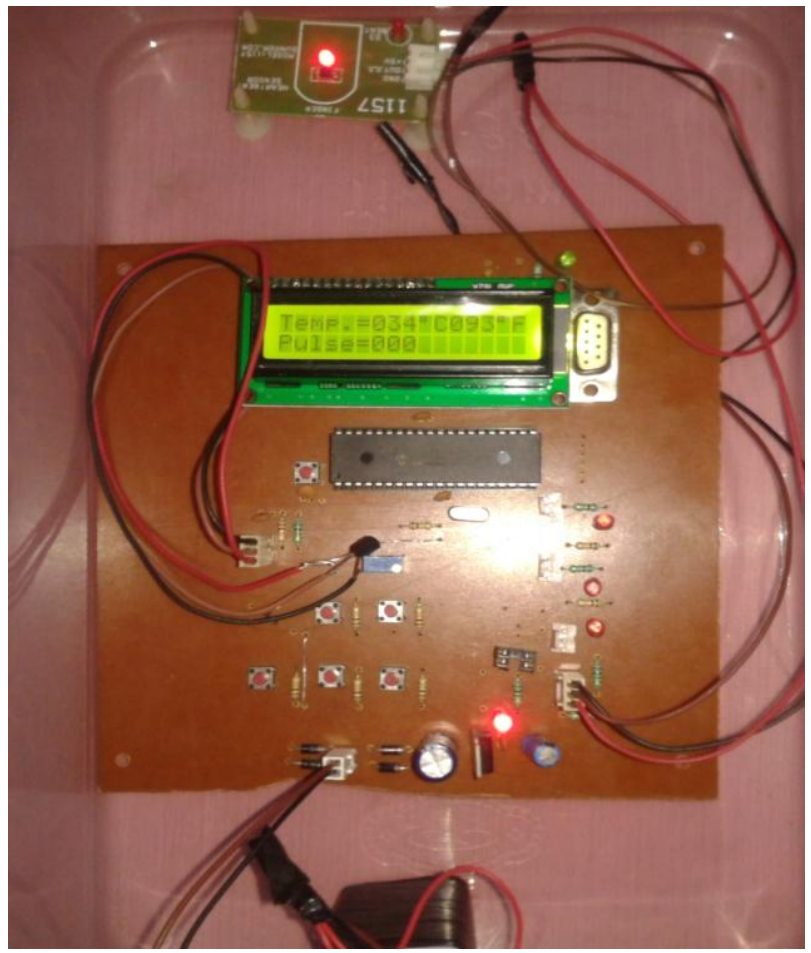

Fig 6-Working Model of Multitasking Human Body Measurement

\section{VI - CONCLUSION}

My project is successfully done all get result but the main challenges include amplifying the desired weak signal in the presence of noise from other muscles and electrical sources. 
Vol. 6 , No. 10, 2021, PP. $54-59$

International Journal of Innovations in Engineering and Science, www.ijies.net

REFERENCES

[1] B.G. Celler; T. Hesketh; W. Earnshaw; E. Ilsar (2002). An instrumentation system for the remote monitoring of changes in functional health status of the elderly at home Baltimore, MD, USA.

[2] Ying Zhang; Hannan Xiao (2009). Bluetooth-Based Sensor Networks for Remotely Monitoring the Physiological Signals of a Patient

[3] Pepijn van de Ven; Alan Bourke; Carlos Tavares; Robert Feld; John Nelson; Artur Rocha; Gearóid Ó Laighin (2010). Integration of a suite of sensors in a wireless health sensor platformChristchurch, New Zealand

[4] Weili Zhou; Jingsheng Liao; Baopu Li; Jingrong Li (2012).A family medical monitoring system based on embedded $u$ C/OS-II and GPRS,Shenyang, China

[5] Daniel Lopez-Martinez; Rosalind Picard (2017). Multi-task neural networks for personalized pain recognition from physiological signals. San Antonio, TX, USA

[6] Neethu Anna Mathew; K M Abubeker (2017). IoT based real time patient monitoring and analysis using Raspberry Pi 3 Chennai, India

[7] Bing Zhang; Jiadong Ren; Yongqiang Cheng; Bing Wang; Zhiyao Wei (2019).Health Data Driven on Continuous Blood Pressure Prediction Based on Gradient Boosting Decision Tree Algorithm

[8] Yue Xu; Yanxin Ji; Fang Deng; Haonan Huang; Qun Hao; Yukun Bao (2019). Wireless Distributed Wearable Health Monitoring System, Xi'an, China

[9] Sheng Ding; Xiaoyan Wang(2020). Medical Remote Monitoring of Multiple Physiological Parameters Based on Wireless Embedded Internet

[10] Sibghat Ullah; Zhao Xu; Hao Wang; Stefan Menzel; Bernhard Sendhoff; Thomas Bäck (2020).Exploring Clinical Time Series Forecasting with Meta-Features in Variational Recurrent Models, Glasgow, UK.

[11] Jianzhong Shang; Christopher J. Payne; James Clark; David P. Noonan; Ka-Wai Kwok; Ara Darzi; Guang-Zhong Yang(2021). Design of a multitasking robotic platform with flexible arms and articulated head for Minimally Invasive Surgery. Vilamoura-Algarve, Portugal 